\title{
HIGHER ORDER COMMUTATORS FOR VECTOR-VALUED CALDERÓN-ZYGMUND OPERATORS
}

\author{
CARLOS SEGOVIA AND JOSÉ L. TORREA
}

\begin{abstract}
Weighted norm estimates for higher order commutators are obtained. The proof, that remain valid in the vector-valued case, are obtained as an application of some extrapolation results. The vector-valued version of the commutator theorem is applied to the Carleson operator, U.M.D. Banach spaces, approximate identities and maximal operators.
\end{abstract}

\section{INTRODUCTION}

The purpose of this paper is to obtain weighted norm estimates for higher order commutators, see Theorem (2.1). The proof of this commutator theorem is an application of some extrapolation results of independent interest stated in $\S 1$. We give proofs that remain valid in the vector-valued case. We apply the vector-valued version of the commutator theorem to the Carleson operator, U.M.D. Banach spaces, approximate identities and maximal operators.

Throughout this paper we shall work on $\mathbf{R}^{n}$ endowed with the Lebesgue measure. Given a Banach space $E$ we shall denote by $L_{E}^{p}\left(\mathbf{R}^{n}\right)$ or simply $L_{E}^{p}$ the Bochner-Lebesgue spaces of $E$-valued strongly measurable functions such that $\int_{R^{n}}\|f(x)\|_{E}^{p} d x<\infty$.

Given a nonnegative measurable function $\alpha(x)$, we shall denote by $L_{E}^{p}(\alpha)$ the space of $E$-valued strongly measurable functions such that

$$
\int_{R^{n}}\|f(x)\|_{E}^{p} \alpha(x) d x<\infty .
$$

By $\mathrm{BMO}_{E}(\nu)$ we mean the space of $E$-valued strongly measurable functions $a(x)$ such that $\int_{Q}\left\|a(x)-a_{Q}\right\|_{E} d x \leq C \int_{Q} \nu(x) d x$ holds for every ball $Q$ and $a_{Q}=|Q|^{-1} \int_{Q} a(x) d x$ with a constant $C$ independent of $Q$.

Given two Banach spaces $E$ and $F$, we denote $\mathscr{L}(E, F)$ the Banach space of all continuous linear operators from $E$ into $F$.

By a Banach lattice we mean an ordered Banach space $F$ over the real numbers satisfying

(i) for every $x$ and $y$ in $F$ there exist a least upper bound $x \vee y$, and a greatest lower bound $x \wedge y$.

(ii) if $|x|=x \vee(-x)$ then $\|x\| \leq\|y\|$, whenever $|x| \leq|y|$. We observe that $\|x\|=\|(|x|)\|$ is a consequence of (ii).

Received by the editors December 8, 1989 and, in revised form, October 15, 1990.

1980 Mathematics Subject Classification (1985 Revision). Primary 42B20, 42B25. 
Let $\alpha(x)$ be a real valued and nonnegative function defined on $\mathbf{R}^{n}$. We shall say that $\alpha$ belongs to the class $A(p, q), 1 \leq p \leq \infty$ and $1 \leq q \leq \infty$, if

$$
\left(|Q|^{-1} \int_{Q} \alpha(x)^{-p^{\prime}} d x\right)^{1 / p^{\prime}}\left(|Q|^{-1} \int_{Q} \alpha(x)^{q}\right)^{1 / q} \leq c
$$

holds for every ball $Q$ with a constant $c$ independent of $Q$. Here $p^{\prime}+p=p \cdot p^{\prime}$. The least constant $c$ satisfying (0.1) shall be called the constant of $\alpha$ in the class $A(p, q)$. The Hardy-Littlewood maximal function $M(f)(x)$ is defined as $\sup \left\{|f|_{Q}: x \in Q\right\}$. It is well known that for $1<p \leq \infty$,

$$
\int|M f(x)|^{p} \omega(x)^{p} d x \leq c_{\omega} \int|f(x)|^{p} \omega(x)^{p} d x
$$

holds if and only if $\omega \in A(p, p)$, see [Mu and RFC]. The "sharp" function of a function $f$ is defined as

$$
f^{\#}(x)=\sup _{x \in Q}|Q|^{-1} \int_{Q}\left\|f(x)-f_{Q}\right\| d x .
$$

As a reference for properties of the maximal function, the sharp function and weights see [RFC].

\section{THE EXTRAPOLATION THEOREM (THE INFINITY CASE)}

We begin this section with a lemma due to Rubio de Francia [RFC, p. 434]. (1.1) Lemma. Let $S$ be an operator, $\rho(x) \geq 0$ and a number $t \geq 1$. Let us assume that

(i) $S(f) \geq 0$,

(ii) $S\left(f_{1}+f_{2}\right) \leq S\left(f_{1}\right)+S\left(f_{2}\right)$,

(iii) $S(\lambda f)=|\lambda| S(f)$, and

(iv) $\|\rho S(f)\|_{t} \leq c\|\rho f\|_{t}$.

Then, if $\rho u$ belongs to $L^{t}$, there exists $U \geq u$ such that

$$
\|\rho U\|_{t} \leq 2\|\rho u\|_{t} \text { and } S(U) \leq 2 c U .
$$

As an application of Rubio de Francia's Lemma, we obtain

(1.2) Proposition. Let $\mu(x)$ and $\eta(x)$ be nonnegative functions. Let $v \geq 1$ and $1<s<\infty$. We assume that, $\mu$ and $\eta \mu$ belong to $A(s, s t)$ where $s^{\prime}>v$ and $s t=s^{\prime} v\left(s^{\prime}-v\right)$. Then if $h(x) \geq 0$ and $h \mu^{s / s^{\prime}}$ belongs to $L^{s^{\prime}}$, there exists $H \geq h$ such that $\left\|H \mu^{s / s^{\prime}}\right\|_{s^{\prime}} \leq 2\left\|h \mu^{s / s^{\prime}}\right\|_{s^{\prime}}$ and both, $\mu^{s} H$ and $\eta \mu^{s} H$, belong to $A(1, v)$.

Proof. Let us define the operator $S$ as

$$
S(f)=\mu^{-s}\left[M\left(\left(\mu^{s} f\right)^{v}\right)\right]^{1 / v}+\eta^{-1} \mu^{-s}\left[M\left(\left(\eta \mu^{s} f\right)\right]^{1 / v} .\right.
$$

This operator $S$ satisfies conditions (i) to (iv) of Lemma (1.1) with $\rho=\mu^{s / s^{\prime}}$ and $t=s^{\prime}$. Then, there exists a function $H \geq h$ such that

$$
\mu^{-s}\left[M\left(\left(\mu^{s} H\right)^{v}\right)\right]^{1 / v} \leq S(H) \leq 2 c H
$$

and

$$
\eta^{-1} \mu^{-s}\left[M\left(\left(\eta \mu^{s} H\right)^{v}\right)\right]^{1 / v} \leq S(H) \leq 2 c H,
$$

showing that $\mu^{s} H$ and $\eta \mu^{s} H$ belong to $A(1, v)$. 
Now, we state and prove the main result of this section. For previous versions of this theorem see [HMS and STa].

(1.3) Theorem (extrapolation theorem, the infinite case). Let $T$ be a sublinear operator defined on $C_{0}^{\infty}, 1 \leq v<\infty, 1<r<\infty, r<p<\lambda \leq \infty, v<$ $(q / r) /(\lambda / r)^{\prime}, q^{-1}=p^{-1}-\lambda^{-1}$. Let $\nu(x) \geq 0$ and assume that

$$
\|\omega T f\|_{\infty} \leq c_{\omega}\|\omega f\|_{\lambda}
$$

holds for every $\omega$ such that $\omega$ and $\nu \omega$ belong to $A\left(\left((\lambda / r)^{\prime} v\right)^{\prime}, \infty\right)$ with a constant $c_{\omega}$ depending on the constants of $\omega$ and $\nu \omega$ in this class. Then, if $\omega^{r}$ and $(\nu \omega)^{r}$ belong to $A\left(\left((p / r)^{\prime} t\right)^{\prime}, q / r\right)$, where $t$ is given by $1 /(q / r)=$ $1 / v(\lambda / r)^{\prime}-1 / t(p / r)^{\prime}$, we have that

$$
\|\omega T f\|_{q} \leq c_{\omega}\|\omega f\|_{p}
$$

hold for every $f \in C_{0}^{\infty}$ with $c_{\omega}$ a constant depending on the constants of $\omega^{r}$ and $(\nu \omega)^{r}$ in their class only.

Moreover, if the hypotheses of the theorem holds for every $v, 1<v<$ $(q / r) /(\lambda / r)^{\prime}$, then if $\omega^{r}$ and $(\nu \omega)^{r}$ belong to $A(p / r, q / r)$ it follows that $(1.5)$ holds for this $\omega$, since there exists $1<v$ close enough to 1 such that $\omega \in$ $A\left(\left((p / r)^{\prime} t\right)^{\prime}, q / r\right)$.

Proof. We can assume without loss of generality that $\|\omega f\|_{p}=1$. Let $h$ be the function defined as

$$
h=\left\{|f| \omega^{(p / r)^{\prime}}\right\}^{r(\lambda / r)^{\prime} p / q} .
$$

This function satisfies

$$
\int h^{(q / r) /(\lambda / r)^{\prime}} \omega^{-r(\lambda / r)^{\prime}\left(1+(p / r)^{\prime} /(q / r)\right)} d x=1
$$

and

$$
\left(\int\left|f h^{-1 / r(\lambda / r)^{\prime}} \omega^{(p / r)^{\prime} /(\lambda / r)^{\prime}}\right|^{\lambda} d x\right)^{1 / \lambda}=1 .
$$

Let $\eta=\nu^{-r(\lambda / r)^{\prime}}, \mu=\omega^{-r(\lambda / r)^{\prime}}$ and $s=1+(p / r)^{\prime} /(q / r)$.

With these notations we have $s^{\prime}=s /(s-1)=(q / r) /(\lambda / r)^{\prime}, s t=s^{\prime} v /\left(s^{\prime}-v\right)$ and $\mu \in A(s, s t)$. Moreover, (1.6) becomes

$$
\left(\int h^{s^{\prime}} \mu^{s} d x\right)^{1 / s^{\prime}}=\left\|h \mu^{s / s^{\prime}}\right\|_{s^{\prime}}=1 .
$$

Then, by Proposition (1.2) there exists $H \geq h$ such that

$$
\left(\int H^{s^{\prime}} \mu^{s}\right)^{1 / s^{\prime}} \leq 2
$$

and

$$
\text { both } \mu^{s} H \text { and } \eta \mu^{s} H \text { belong to } A(1, v) \text {. }
$$

Let $G=H^{-1 / r(\lambda / r)^{\prime}} \omega^{(p / r)^{\prime} /(\lambda / r)^{\prime}}$. We shall prove that $G^{r}$ and $(\nu G)^{r}$ belong to $A\left(\left((\lambda / r)^{\prime} v\right)^{\prime}, \infty\right)$ or, equivalently, that $G^{-r(\lambda / r)^{\prime}}$ and $(\nu G)^{-r(\lambda / r)^{\prime}}$ belong to 
$A(1, v)$. In fact, since $r(\lambda / r)^{\prime} s=r(p / r)^{\prime},(1.9)$ tells us that

$$
G^{-r(\lambda / r)^{\prime}}=H \omega^{-r(p / r)^{\prime}}=\mu^{s} H \in A(1, v)
$$

and

$$
(\nu G)^{-r(\lambda / r)^{\prime}}=H \nu^{-r(\lambda / r)^{\prime}} \omega^{-r(p / r)^{\prime}}=\eta \mu^{s} H \in A(1, v) .
$$

Now, recalling (1.7), we get

$$
1 \geq\left(\int\left|f H^{-1 / r(\lambda / r)^{\prime}} \omega^{(p / r)^{\prime} /(\lambda / r)^{\prime}}\right|^{\lambda} d x\right)^{1 / \lambda}=\left(\int|f G|^{\lambda}\right)^{1 / \lambda} .
$$

Then, by (1.10), (1.11), (1.8) and (1.4), it follows that

$$
\begin{aligned}
1 & \geq c_{\omega}^{-1}\|G T f\|_{\infty} c\left(\int H^{s^{\prime}} \mu^{s} d x\right)^{1 / q} \\
& \geq c\left(\int G^{q} H^{s^{\prime}} \mu^{s}|T f|^{q} d x\right)^{1 / q} \geq c\left(\int \omega^{q}|T f|^{q} d x\right)^{1 / q},
\end{aligned}
$$

as we wanted to show.

\section{COMMUTATORS FOR FRACTIONAL AND SINGULAR INTEGRALS}

In this section we state the main results of this paper.

(2.1) Theorem (Commutator theorem). Let $E$ and $F$ be Banach spaces and $K$ a bounded linear operator from $L_{E}^{p}\left(R^{n}\right)$ into $L_{F}^{q}\left(R^{n}\right), q^{-1}=p^{-1}-\lambda^{-1}$, $1<p<\lambda$. Let us assume that there exists a kernel $k(x, y)$ with values in $\mathscr{L}(E, F)$, such that

(2.2) if $f \in C_{0}^{\infty}$, then

$$
K f(x)=\int k(x, y) f(y) d y \quad \text { if } x \notin \operatorname{supp} f,
$$

(2.3) there exists a slowly increasing function $\omega(t) \geq 0 \quad(\omega(2 t) \leq c \omega(t))$ such that

$$
\begin{aligned}
& \left(\int_{R<\left|y-y_{0}\right|<2 R}\|k(x, y)-k(z, y)\|^{r^{\prime}} d y\right)^{1 / r^{\prime}} \\
& \quad \leq c R^{-n / r(\lambda / r)^{\prime}}\left(|x-z| / R+\int_{|x-z| / 2 R<t<|x-z| / R} \omega(t) d t / t\right) .
\end{aligned}
$$

Let us assume that two functions $a(x)$ and $\tilde{a}(x)$ defined on $R^{n}$ and with values in $\mathscr{L}(E, E)$ and $\mathscr{L}(F, F)$, respectively, are given and satisfy

(i) for every $x$ and $y$ in $R^{n}$,

$$
a(x) a(y)=a(y) a(x) \quad \text { and } \quad \tilde{a}(x) \tilde{a}(y)=\tilde{a}(y) \tilde{a}(x)
$$

hold,

(ii) for every $z \in R^{n}$,

$$
\tilde{a}(z) K(f)(x)=K(a(z) f)(x)
$$

holds, and 
(iii) a belongs to $\mathrm{BMO}_{\mathscr{L}(E, E)}(\nu)$ and ã belongs to $\mathrm{BMO}_{\mathscr{L}(F, F)}(\nu)$.

We define the m-commutator $C_{a, \tilde{a}}^{m}(f)$ as

$$
\begin{aligned}
& C_{a, \tilde{a}}^{0}(f)(x)=K f(x) \text { and, if } m>0, \\
& C_{a, \tilde{a}}^{m}(f)(x)=\tilde{a}(x) C_{a, \tilde{a}}^{m-1}(f)(x)-C_{a, \tilde{a}}^{m-1}(a f)(x) .
\end{aligned}
$$

Then, given $\alpha$ and $\beta$ such that $\alpha^{r}$ and $\beta^{r}$ belong to $A(p / r, q / r), 1 \leq r<$ $p<\lambda \leq \infty, q^{-1}=p^{-1}-\lambda^{-1}$ and $\alpha \beta^{-1}=\nu^{m}$ there exists a finite constant $c$ such that

$$
\left(\int\left\|C_{a, \tilde{a}}^{m}(f)(x)\right\|_{F}^{q} \beta(x)^{q} d x\right)^{1 / q} \leq c\left(\int\|f\|_{E}^{p} \alpha(x)^{p} d x\right)^{1 / p}
$$

holds provided that the Dini condition

$$
\int_{0}^{1} \lg ^{m}(1 / t) \omega(t) d t / t<\infty
$$

is satisfied.

For the case $m=0$ this theorem is essentially due to Kurtz and Wheeden, see [KW]. For any $m \geq 1$ and $\nu=1$, see [CRW]. For $m=1$ and the Hilbert transform, see [B].

(2.4) Theorem. Let $F$ be a Banach lattice and $W(f)$ a bounded linear operator from $L^{p}\left(R^{n}\right)$ into $L_{F}^{q}\left(R^{n}\right), 1<p<\lambda \leq \infty, q^{-1}=p^{-1}-\lambda^{-1}$. Let us assume the existence of an $F$-valued kernel $w(x, y)$ such that

$\left(\mathrm{W}_{1}\right)$ for every $f \in C_{0}^{\infty}$,

$$
W(f)(x)=\int w(x, y) f(y) d y
$$

holds,

$\left(\mathrm{W}_{2}\right)$ for every $x$ and $y$ in $R^{n}, w(x, y) \geq 0$, and

$\left(\mathrm{W}_{3}\right)$ the kernel $w(x, y)$ satisfies condition (2.3), substituting $k(x, y)$ for $w(x, y)$.

Let us assume that a real valued function $a(x)$ is given and belongs to $\mathrm{BMO}(\nu)$. Then, the operator

$$
W_{a}^{m}(f)(x)=\int w(x, y)|a(x)-a(y)|^{m} f(y) d y,
$$

is well defined on $C_{0}^{\infty}$ and there exists a finite constant $c$ such that

$$
\left(\int\left\|W_{a}^{m}(f)(x)\right\|^{q} \beta(x)^{q} d x\right)^{1 / q} \leq c\left(\int|f(y)|^{p} \alpha(x)^{p} d x\right)^{1 / p}
$$

holds whenever $\alpha^{r}$ and $\beta^{r}$ belong to $A(p / r, q / r), 1 \leq r<p<\lambda \leq \infty$, $q^{-1}=p^{-1}-\lambda^{-1}$, and $\alpha \beta^{-1}=\nu^{m}$, provided that

$$
\int_{0}^{1} \lg ^{m}(1 / t) \omega(t) d t / t<\infty
$$




\section{Applications}

A. Singular integrals and fractional integrals. Let $\Omega(x)$ be a homogeneous function of degree zero defined on $R^{n}-\{0\}$. We assume that $\int_{\left|x^{\prime}\right|=1} \Omega\left(x^{\prime}\right) d x^{\prime}=$ 0 and if $\rho$ is a rotation in $R^{n}$ and $|\rho|=\|\rho-I\|$, let

$$
\sup _{|\rho|<t}\left(\int_{\left|x^{\prime}\right|=1}\left|\Omega\left(\rho x^{\prime}\right)-\Omega\left(x^{\prime}\right)\right|^{r^{\prime}} d x^{\prime}\right)^{1 / r^{\prime}}=\omega(t), \quad 1 \leq r<\infty .
$$

We assume that $\omega(t)$ satisfies the condition

$$
\int_{0}^{1} \lg ^{m}(1 / t) \omega(t) d t / t<\infty
$$

Then, by $[\mathrm{KW}]$ we obtain that the kernel $k(x-y)=\Omega(x-y)|x-y|^{-n / \lambda^{\prime}}$, $1<\lambda \leq \infty$, satisfies $(2.3)$ and

$$
K f(x)=\text { p.v. } \int \frac{\Omega(x-y)}{|x-y|^{n / \lambda^{\prime}}} \cdot f(y) d y,
$$

is a bounded operator from $L^{p}$ into $L^{q}$ for every $1<p<\lambda, q^{-1}=p^{-1}-\lambda^{-1}$. Thus, by the commutator theorem (2.1) we obtain that if $a \in \operatorname{BMO}(\nu)$, then

$$
C_{a}^{m}(f)(x)=\text { p.v. } \int \frac{\Omega(x-y)}{|x-y|^{n / \lambda^{\prime}}}(a(x)-a(y))^{m} f(y) d y,
$$

satisfies

$$
\left\|\beta C_{a}^{m} f\right\|_{q} \leq c\|\alpha f\|_{p},
$$

for every $\alpha$ and $\beta, \alpha \beta^{-1}=\nu^{m}, \alpha^{r}$ and $\beta^{r}$ in $A(p / r, q / r)$ and $q^{-1}=$ $p^{-1}-\lambda^{-1}, 1 \leq r<p<\lambda \leq \infty$. For the case of the Hilbert transform and $m=1$ see [B]. For kernels with condition (3.1) and $m=1$, see [STa and STb]. Finally, the unweighted case was considered in [CH and CRW].

B. Let us assume that $k(x, y)$ is a kernel satisfying condition $(2.3)$ and that

$$
K f(x)=\lim _{\varepsilon \rightarrow 0} \int_{|x-y|>\varepsilon} k(x, y) f(y) d y,
$$

defines a bounded linear operator from $L^{p}$ into $L^{q}, 1<p<\lambda, q^{-1}=$ $p^{-1}-\lambda^{-1}$. Then, if $a \in \mathrm{BMO}(\nu)$,

$$
\left(C_{a}^{m}\right)^{*}(f)(x)=\sup _{\varepsilon>0}\left|\int_{|x-y|>\varepsilon} k(x, y)(a(x)-a(y))^{m} f(y) d y\right|,
$$

is a bounded linear operator from $L^{p}\left(\alpha^{p}\right)$ into $L^{q}\left(\beta^{q}\right)$ provided that $\alpha^{r}$ and $\beta^{r}$ belong to $A(p / r, q / r), 1 \leq r<p<\lambda \leq \infty, q^{-1}=p^{-1}-\lambda^{-1}$, and $\alpha \beta^{-1}=\nu^{m}$. In fact let $\phi$ and $\psi$ be $C^{\infty}$ functions such that

$$
\chi_{[2, \infty)} \leq \phi \leq \chi_{[1, \infty)} \quad \text { and } \chi_{[1,2]} \leq \psi \leq \chi_{[1 / 2,3]} .
$$

We consider the $l^{\infty}$-valued operators

$$
\Phi(f)(x)=\left(\int k(x, y) \phi(|x-y| / \varepsilon) f(y) d y\right)_{\varepsilon>0}
$$

and 


$$
\Psi(f)(x)=\left(\int|k(x, y)| \psi(|x-y| / \varepsilon) f(y) d y\right)_{\varepsilon>0} .
$$

The kernels of $\Phi$ and $\Psi$ satisfy conditions (2.3) and $\left(W_{3}\right)$ of (2.4) respectively. By the vector valued theory of the Calderón-Zygmund operators, see [FRT and $\mathrm{RT}]$, these operators are bounded from $L^{p}$ into $L_{l \infty}^{q}, 1<p<\lambda \leq \infty, q^{-1}=$ $p^{-1}-\lambda^{-1}$. Therefore, $\Phi$ satisfies the hypotheses of Theorem $(2.1)$ and $\Psi$ those of Theorem (2.4). Let $a(x)$ be in $\mathrm{BMO}(\nu)$ and $\tilde{a}(x)$ in $\mathrm{BMO}_{l \infty}(\nu)$ defined as $\tilde{a}_{\varepsilon}(x)=a(x)$. Then

$$
\Phi_{a}^{m}(f)(x)=\left(\int k(x, y)(a(x)-a(y))^{m} \phi(|x-y| / \varepsilon) f(y) d y\right)_{\varepsilon}
$$

and

$$
\Psi_{a}^{m}(f)(x)=\left(\int|k(x, y)||a(x)-a(y)|^{m} \psi(|x-y| / \varepsilon) f(y) d y\right)_{\varepsilon},
$$

are bounded linear operators from $L^{p}\left(\alpha^{p}\right)$ into $L_{l \infty}^{q}\left(\beta^{q}\right)$ for $1 \leq r<p<\lambda \leq$ $\infty, q^{-1}=p^{-1}-\lambda^{-1}, \alpha \beta^{-1}=\nu^{m}, \alpha^{r}$ and $\beta^{r}$ in $A(p / r, q / r)$. If we define

$$
\widehat{C}_{a}^{m}(f)(x)=\left(\int_{|x-y|>\varepsilon} k(x, y)(a(x)-a(y))^{m} f(y) d y\right)_{\varepsilon>0},
$$

then, the difference

$$
\begin{aligned}
& U_{a}^{m}(f)(x)=\Phi_{a}^{m}(f)(x)-\widehat{C}_{a}^{m}(f)(x) \\
& \quad=\left(\int k(x, y)(a(x)-a(y))^{m}\left\{\phi(|x-y| / \varepsilon)-\chi_{[1, \infty)}(|x-y| / \varepsilon)\right\} f(y) d y\right)_{\varepsilon>0},
\end{aligned}
$$

satisfies

$$
\begin{aligned}
\left\|U_{a}^{m} f(x)\right\|_{l \infty} & \leq \sup _{\varepsilon>0}\left(\int|k(x, y)||a(x)-a(y)|^{m} \psi(|x-y| / \varepsilon)|f(y)| d y\right) \\
& =\left\|\Psi_{a}^{m}(f)(x)\right\|_{l \infty} .
\end{aligned}
$$

Therefore, $U_{a}^{m}(f)$ is a bounded operator and this shows that $\widehat{C}_{a}^{m}(f)$ is bounded under the same conditions. Thus,

$$
\left(C_{a}^{m}\right)^{*}(f)(x)=\left\|\widehat{C}_{a}^{m}(f)(x)\right\|_{l \infty}
$$

is a bounded operator.

C. Let

$$
S f(x)=\left\{\int \frac{e^{-i r y}}{x-y} f(y) d y\right\}_{r \in \mathbf{R}} .
$$

Since, by the Carleson-Hunt theorem, see $[\mathrm{H}]$, this operator is bounded from $L^{p}$ into $L_{l \infty}^{p}, 1<p<\infty$, and observing that the kernel $k(x, y)=e^{-i r y} /(x-y)$ satisfies the conditions of Theorem (2.1) then

$$
S_{a}^{m}(f)=\left\{\int \frac{e^{-i r y}}{x-y}(a(x)-a(y))^{m} f(y) d y\right\}_{r \in \mathbf{R}},
$$

is a bounded operator from $L^{p}\left(\alpha^{p}\right)$ into $L_{l \infty}^{p}\left(\beta^{p}\right)$, provided that $1<p<\infty$, $\alpha$ and $\beta$ belong to $A(p, p)$, and $\alpha \beta^{-1}=\nu^{m}$, and $a$ belongs to BMO. 
D. Let $E$ be an U.M.D. space. Then, the Hilbert transform

$$
H f(x)=\text { p.v. } \int f(y) /(x-y) d y,
$$

is known to be a bounded linear operator from $L_{E}^{p}$ into $L_{E}^{p}$, see [Bu]. Therefore, by Theorem (2.1) we get that

$$
C_{a}^{m}(f)(x)=\text { p.v. } \int(a(x)-a(y))^{m} f(y) /(x-y) d y,
$$

is a bounded linear operator from $L_{E}^{p}\left(\alpha^{p}\right)$ into $L_{E}^{p}\left(\beta^{p}\right), \alpha$ and $\beta$ in $A(p, p)$, $\alpha \beta^{-1}=\nu^{m}, 1<p<\infty, a$ belongs to $\mathrm{BMO}_{\mathscr{L}(E, E)}$ and satisfies $a(x) a(y)=$ $a(y) a(x)$ for every $x$ and $y$.

E. Maximal operators. Let $0 \leq \phi \in L^{1}\left(\mathbf{R}^{n}\right)$ verify $|\phi(x-y)-\phi(x)| \leq$ $c|y| /|x|^{n+1}$, provided that $|x|>2|y|$. Set $\phi_{\varepsilon}(x)=\varepsilon^{-n / \lambda^{\prime}} \phi(x / \varepsilon)$. Then the operator $M_{\phi}(f)(x)=\left(f * \phi_{\varepsilon}(x)\right)_{\varepsilon>0}$, is a bounded linear operator from $L^{p}$ into $L_{l \infty}^{q}$, see [FRT and RT]. It is easy to check that the kernel $w(x, y)=\phi_{\varepsilon}(|x-y|)$ satisfies the hypotheses of Theorem (2.4). Therefore, if $a \in \operatorname{BMO}(\nu)$, the operator

$$
M_{\phi, a}^{m}(f)(x)=\left(\int|a(x)-a(y)|^{m} \phi_{\varepsilon}(x-y) f(y) d y\right)_{\varepsilon>0},
$$

is a bounded operator from $L^{p}\left(\alpha^{p}\right)$ into $L_{l \infty}^{q}\left(\beta^{q}\right), 1<p<\lambda \leq \infty, q^{-1}=$ $p^{-1}-\lambda^{-1}, \alpha$ and $\beta$ in $A(p, q), \alpha \beta^{-1}=\nu^{m}$, meaning that the operator

$$
\sup _{\varepsilon>0} \int|a(x)-a(y)|^{m} \phi_{\varepsilon}(x-y) f(y) d y,
$$

is bounded from $L^{p}\left(\alpha^{p}\right)$ into $L^{q}\left(\beta^{q}\right)$ under the same conditions on $p, q, \lambda, \alpha$, $\beta$ and $\nu$. In particular taking $\phi$ such that $\chi_{[-1,1]} \leq \phi$, we obtain that

$$
S_{a}^{m}(f)(x)=\sup _{x \in Q}|Q|^{-1 / \lambda^{\prime}} \int_{Q}|a(x)-a(y)|^{m} f(y) d y,
$$

is a bounded linear operator from $L^{p}\left(\alpha^{p}\right)$ into $L^{q}\left(\beta^{q}\right)$. Moreover we have the following theorem:

(3.2) Theorem. Let $\nu$ be such that $\nu^{m \lambda^{\prime} / 2} \in A(2,2)$, then the following conditions are equivalent:

(a) For every $p$ and $q$ such that $q^{-1}=p^{-1}-\lambda^{-1}, \alpha$ and $\beta$ in $A(p, q)$ and $\alpha \beta^{-1}=\nu^{m}$, the operator $S_{a}^{m}(f)$ maps $L^{p}\left(\alpha^{p}\right)$ into $L^{q}\left(\beta^{q}\right)$.

(b) The function $a(x)$ belongs to $\mathrm{BMO}(\nu)$.

Proof. We have seen that (b) implies (a). Let us prove that (a) implies (b). By the factorization theorem of weights, see [RFC], there exists $\nu_{0}$ and $\nu_{1}$ such that $\nu^{m \lambda^{\prime}}=\nu_{0} \nu_{1}^{-1}, \nu_{0}$ and $\nu_{1}$ belonging to $A(1,1)$. Let $p=2 \lambda /(\lambda+1)$, $q=2 \lambda^{\prime}$, then $1<p<\lambda, p^{\prime}=q$, and $q^{-1}=p^{-1}-\lambda^{-1}$. Let $\alpha=\left(\nu_{0} \nu_{1}^{-1}\right)^{1 / q}$ and $\beta=\left(\nu_{0}^{-1} \nu_{1}\right)^{1 / q}$, then $\alpha \beta^{-1}=\nu^{m}$ and $\alpha$ and $\beta$ belong to $A(p, q)$. 
Therefore, we have

$$
\begin{aligned}
& \left(|Q|^{-1} \int_{Q}\left|a(x)-a_{Q}\right| d x\right)^{m} \leq|Q|^{-1} \int_{Q}\left|a(x)-a_{Q}\right|^{m} d x \\
& \leq|Q|^{-2} \int_{Q}\left(\int_{Q}|a(x)-a(y)|^{m} d y\right) d x \\
& \leq|Q|^{-1 / p}\left(\int_{Q}\left(|Q|^{-1 / \lambda^{\prime}} \int_{Q}|a(x)-a(y)|^{m} d y\right)^{q} \beta^{q}(x) d x\right)^{1 / q} \\
& \cdot\left(|Q|^{-1} \int_{Q} \beta^{-q^{\prime}} d x\right)^{1 / q^{\prime}} \\
& \leq|Q|^{-1 / p}\left(\int S_{a}^{m}\left(\chi_{Q}\right)^{q} \beta^{q} d x\right)^{1 / q}\left(|Q|^{-1} \int_{Q} \beta^{-q^{\prime}} d x\right)^{1 / q^{\prime}} \\
& \leq c\left(|Q|^{-1} \int_{Q} \alpha^{p} d x\right)^{1 / p}\left(|Q|^{-1} \int_{Q} \beta^{-q^{\prime}} d x\right)^{1 / q^{\prime}} .
\end{aligned}
$$

Since $\alpha^{p}=\beta^{-q^{\prime}}=\nu^{m \lambda /(\lambda+1)}, p=q^{\prime}=2 \lambda /(\lambda+1)$ we get

$$
\left(|Q|^{-1} \int_{Q}\left|a(x)-a_{Q}\right| d x\right)^{m} \leq c\left(|Q|^{-1} \int_{Q} \nu^{m} d x\right) .
$$

Then, by Lemma (4.4) in the following section, we obtain

$$
|Q|^{-1} \int_{Q}\left|a(x)-a_{Q}\right| d x \leq c|Q|^{-1} \int_{Q} \nu(x) d x
$$

as claimed.

\section{PROOFS OF THE COMMUTATOR THEOREMS}

We begin this section with a sequence of lemmas.

(4.1) Lemma. Let a belong to $\operatorname{BMO}(\nu), \alpha \beta^{-1}=\nu^{m}, m \geq 1, \alpha$ and $\beta$ in $A\left(s^{\prime}, \infty\right), 1 \leq s<\infty$. Then

$$
\left(|Q|^{-1} \int_{Q}\left\|a(x)-a_{Q}\right\|^{m s} \alpha(x)^{-s}\right)^{1 / s} \leq c\left(\inf _{Q} \beta^{-1}\right)
$$

holds for every ball $Q$ with a constant $c$ depending on the class constants of $\alpha$ and $\beta$ only.

For a proof of this lemma see [B].

(4.2) Lemma. If $\nu^{m} \in A_{\infty}$ and satisfies

$$
|Q|^{-1} \int_{Q} \nu^{m(1+\varepsilon)}(x) d x \leq c\left(|Q|^{-1} \int_{Q} \nu^{m}(x) d x\right)^{1+\varepsilon}
$$

for every ball $Q ; c$ independent of $Q$, then

$$
|Q|^{-1} \int_{Q} \nu(x)^{m(1+\varepsilon)} d x \leq c\left(|Q|^{-1} \int_{Q} \nu(x) d x\right)^{m(1+\varepsilon)}
$$

for every ball $Q, c$ independent of $Q$.

For a proof see [STW]. 
(4.4) Lemma. If $a \in \mathrm{BMO}(\nu)$ and (4.3) holds, then

$$
\left(|Q|^{-1} \int_{Q}\left\|a(x)-a_{Q}\right\|^{m(1+\varepsilon)} d x\right) \leq\left(|Q|^{-1} \int_{Q} \nu(x) d x\right)^{m(1+\varepsilon)}
$$

holds for every ball $Q$ with $c$ independent of $Q$.

For a proof see [B].

(4.5) Lemma. Let $\alpha$ and $\beta$ be weights such that $\alpha^{r}$ and $\beta^{r}$ belong to $A\left(\left((p / r)^{\prime} t\right)^{\prime}, q\right), t \geq 1$, and $\alpha \beta^{-1}=\nu^{m}, m \geq 1$. Then, if $\delta_{k}=\alpha^{(1-k / m)} \beta^{k / m}$, $0 \leq k \leq m$, we have

$$
\delta_{k}^{r} \in A\left(\left((p / r)^{\prime} t\right)^{\prime}, q\right) \text { and } \delta_{k} \delta_{h}^{-1}=\nu^{h-k}, \quad \text { if } 0 \leq k<h \leq m .
$$

Proof. We have

$$
|Q|^{-1} \int_{Q} \delta^{-r(p / r)^{\prime} t} d x=|Q|^{-1} \int_{Q} \alpha^{-r(p / r)^{\prime} t(1-k / m)} \beta^{-r(p / r)^{\prime} t k / m} d x
$$

Then, by Hölder inequality, we get that the integral above is smaller than or equal to

$$
\left(|Q|^{-1} \int_{Q} \alpha^{-r(p / r)^{\prime} t} d x\right)^{(1-k / m)}\left(|Q|^{-1} \int_{Q} \beta^{-r(p / r)^{\prime} t} d x\right)^{k / m} .
$$

Analogously,

$$
|Q|^{-1} \int_{Q} \delta_{k}^{q} d x \leq\left(|Q|^{-1} \int_{Q} \alpha^{q} d x\right)^{(1-k / m)}\left(|Q|^{-1} \int_{Q} \beta^{q} d x\right)^{k / m}
$$

and the lemma is proved.

(4.6) Lemma. Let $K$ be the operator in the statement of Theorem (2.1). Let $Q$ be $a$ ball and $f$ a function with support contained in $4 Q$. Then if $\alpha, \beta$, and $\nu$ are weights such that $\alpha \beta^{-1}=\nu^{m}, m \geq 1, \alpha^{r}$ and $\beta^{r}$ belong to $A\left(\left((\lambda / r)^{\prime} v\right)^{\prime}, \infty\right), v>1$, we have

$$
\begin{aligned}
|Q|^{-1} & \int_{Q}\left\|K\left(\left(a-a_{Q}\right)^{m} f\right)(z)\right\| d x \\
& \leq c\left(\inf _{Q} \beta^{-1}\right)\left(\int\|f(y)\|^{\lambda} \alpha(y)^{\lambda} d y\right)^{1 / \lambda},
\end{aligned}
$$

where the constant $c$ depends on the class constants of $\alpha$ and $\beta$ only.

If $W$ is the operator in the statement of Theorem (2.4), we have that

$$
\begin{aligned}
|Q|^{-1} & \int_{Q}\left\|W\left(\left|a-a_{Q}\right|^{m}|f|\right)(z)\right\| d z \\
& \leq c\left(\inf _{Q} \beta^{-1}\right)\left(\int\|f(y)\|^{\lambda} \alpha(y)^{\lambda} d y\right)^{1 / \lambda}
\end{aligned}
$$

holds with the same hypotheses as before. 
Proof. Let $p$ be defined by $p(\lambda / p)^{\prime}=r(\lambda / r)^{\prime} v$. Then $r<p<\lambda$ and if $1 / q=1 / p-1 / \lambda$ we get

$$
\begin{aligned}
|Q|^{-1} & \int_{Q}\left\|K\left(\left(a-a_{Q}\right)^{m} f\right)(z)\right\| d z \\
\leq & |Q|^{-1 / q}\left(\int_{Q}\left\|K\left(\left(a-a_{Q}\right)^{m} f\right)(z)\right\|^{q} d z\right)^{1 / q} \\
\leq & c|Q|^{-1 / q}\left(\int_{4 Q}\left(\left\|a(y)-a_{Q}\right\|^{m}\|f(y)\|\right)^{p} d y\right)^{1 / p} \\
\leq & c|Q|^{-1 / q}\left(\int_{4 Q}\left\|a(y)-a_{Q}\right\|^{m p(\lambda / p)^{\prime}} \alpha(y)^{-p(\lambda / p)^{\prime}} d y\right)^{1 / p(\lambda / p)^{\prime}} \\
& \cdot\left(\int\|f(y)\|^{\lambda} \alpha(y)^{\lambda}\right)^{1 / \lambda} .
\end{aligned}
$$

Since $q=p(\lambda / p)^{\prime}$ and applying Lemma (4.1) we obtain that (4.7) is bounded by $c\left(\inf _{Q} \beta^{-1}\right)\left(\int\|f(y)\|^{\lambda} \alpha(y)^{\lambda} d y\right)^{1 / \lambda}$, as we wanted to show.

As for (4.8), the proof is the same.

(4.9) Lemma. Under the same hypotheses of Lemma (4.6), we have

$$
\begin{aligned}
& |Q|^{-1} \int_{Q}\left\|\tilde{a}(x)-\tilde{a}_{Q}\right\|^{m}\|K f(x)\| d x \\
& \quad \leq c\left(\inf _{Q} \beta^{-1}\right)\left(\int\|f(x)\|^{\lambda} \alpha(x)^{\lambda} d x\right)^{1 / \lambda},
\end{aligned}
$$

and

$$
\begin{aligned}
& |Q|^{-1} \int_{Q}\left|a(x)-a_{Q}\right|^{m}\|W(f)(x)\| d x \\
& \quad \leq\left(\inf _{Q} \beta^{-1}\right)\left(\int\|f(x)\|^{\lambda} \alpha(x)^{\lambda} d x\right)^{1 / \lambda} .
\end{aligned}
$$

Proof. Let us choose $\varepsilon, 0<\varepsilon<1$, such that

(i) $r<\lambda(1-\varepsilon)$.

(ii) if $s=1+\varepsilon /((1-\varepsilon) \lambda-\varepsilon)$, then $s<r(\lambda / r)^{\prime} v$.

(iii) $(\lambda(1-\varepsilon) / r)^{\prime} \leq(\lambda / r)^{\prime} v$.

Then, let $p=\lambda(1-\varepsilon)$. Then, by (i) we have $r<p<\lambda$ and $1 / s^{\prime}=1 / p-1 / \lambda$.

Thus, the first member of (4.10) is less than or equal to

$$
\left(|Q|^{-1} \int_{Q}\left\|\tilde{a}(x)-\tilde{a}_{Q}\right\|^{m s} \alpha(x)^{-s} d x\right)^{1 / s}\left(|Q|^{-1} \int\|K(f)(x)\|^{s^{\prime}} \alpha(x)^{s^{\prime}} d x\right)^{1 / s^{\prime}} \text {. }
$$

Since by (iii), $r(p / r)^{\prime} \leq r(\lambda / r)^{\prime} v$, we have

$$
\begin{gathered}
\left(|Q|^{-1} \int_{Q} \alpha^{-r(p / r)^{\prime}} d x\right)^{1 /(p / r)^{\prime}}\left(|Q|^{-1} \int_{Q} \alpha^{q}\right)^{1 /(q / r)} \\
\leq c\left(\inf _{Q} \alpha^{-1}\right)^{r}\left(|Q|^{-1} \int_{Q} \alpha^{q}\right)^{r / q} \leq c
\end{gathered}
$$


showing that $\alpha^{r}$ belong to $A(p / r, q / r)$. Thus

$$
\begin{aligned}
& \left(|Q|^{-1} \int\|K(f)(x)\|^{s^{\prime}} \alpha(x)^{s^{\prime}} d x\right)^{1 / s^{\prime}} \\
& \quad \leq c|Q|^{-1 / s^{\prime}}\left(\int_{4 Q}\|f(x)\|^{p} \alpha(x)^{p} d x\right)^{1 / p} \\
& \quad \leq c|Q|^{-1 / s^{\prime}+1 / p(\lambda / p)^{\prime}}\left(\int\|f(x)\|^{\lambda} \alpha(x)^{\lambda} d x\right)^{1 / \lambda} \\
& \quad=c\left(\int\|f(x)\|^{\lambda} \alpha(x)^{\lambda} d x\right)^{1 / \lambda} .
\end{aligned}
$$

Since by (ii) we know that $s<r(\lambda / r)^{\prime} v$, then by Lemma (4.1)

$$
\left(|Q|^{-1} \int_{Q}\left\|\tilde{a}(x)-\tilde{a}_{Q}\right\|^{m s} \alpha(x)^{-s} d x\right) \leq c\left(\inf _{Q} \beta^{-1}\right) .
$$

Therefore, (4.10) holds as claimed.

As for (4.11) the proof is the same. We observe that the lemma is true if $m=0$. The proof is simple.

(4.12) Lemma. Let $a \in \operatorname{BMO}(\nu), \delta \beta^{-1}=\nu^{k}, k \geq 0, \delta$ and $\beta$ belonging to $A\left(v^{\prime}, \infty\right), v>1$. Then

$$
\left(|Q|^{-1} \int_{Q}\left|a-a_{Q}\right|^{k} d x\right)\left(|Q|^{-1} \int_{Q}|g|\right) \leq c\left(\inf _{Q} \beta^{-1}\right)\|\delta g\|_{\infty}
$$

holds, with a constant $c$ depending on the class constants of $\delta$ and $\beta$ only.

Proof. We have

$$
|Q|^{-1} \int_{Q}|g| d x \leq\|g \delta\|_{\infty}\left(|Q|^{-1} \int_{Q} \delta^{-1} d x\right) \leq c\|g \delta\|_{\infty}\left(\inf _{Q} \delta^{-1}\right) .
$$

Therefore, the first member of $(4.13)$ is bounded by

$$
c\left(|Q|^{-1} \int_{Q}\left|a-a_{Q}\right|^{k} \delta^{-1}\right)\|g \delta\|_{\infty} \leq c\left(|Q|^{-1} \int_{Q}\left|a-a_{Q}\right|^{k v} \delta^{-v}\right)^{1 / v}\|g \delta\|_{\infty},
$$

and applying Lemma (4.1) we get (4.13).

(4.14) Lemma. Let $k(x, y)$ and $w(x, y)$ be kernels satisfying the hypotheses of Theorems (2.1) and (2.4). Assume that the support of $f(x)$ is contained in $C 4 Q$. Let $\alpha^{r}$ and $\beta^{r}$ belong to $A(\lambda / r, \infty), \alpha \beta^{-1}=\nu^{m}, m \geq 0, a$ and $\tilde{a} \in \operatorname{BMO}(\nu)$. Then,

$$
\begin{aligned}
& \text { if } z \in Q \text {, we have } \\
& \begin{aligned}
|Q|^{-1} & \int_{Q}\left\|\tilde{a}(x)-\tilde{a}_{Q}\right\|^{m}\left(\int\|k(x, y)-k(z, y)\|\|f(y)\| d y\right) d x \\
& \leq c\left(\inf _{Q} \beta^{-1}\right)\left(\int\|f\|^{\lambda} \alpha^{\lambda} d x\right)^{1 / \lambda},
\end{aligned}
\end{aligned}
$$


if $x, z \in Q$, then

$$
\begin{gathered}
\int\|k(x, y)-k(z, y)\|\left\|a(y)-a_{Q}\right\|^{m}\|f(y)\| d y \\
\leq c\left(\inf _{Q} \beta^{-1}\right)\left(\int\|f\|^{\lambda} \alpha^{\lambda} d x\right)^{1 / \lambda}
\end{gathered}
$$

if $z \in Q$, we have

$$
\begin{aligned}
& |Q|^{-1} \int_{Q}\left|a(x)-a_{Q}\right|^{m}\left(\int\|w(x, y)-w(z, y)\||f(y)| d y\right) d x \\
& \leq c\left(\inf _{Q} \beta^{-1}\right)\left(\int|f|^{\lambda} \alpha^{\lambda} d x\right)^{1 / \lambda}, \text { and } \\
& \text { if } x, z \in Q, \text { then } \\
& \quad \int\|w(x, y)-w(z, y)\|\left|a(y)-a_{Q}\right|^{m}|f(y)| d y \\
& \leq \leq\left(\inf _{Q} \beta^{-1}\right)\left(\int|f|^{\lambda} \alpha^{\lambda} d x\right)^{1 / \lambda}
\end{aligned}
$$

hold with a constant $c$ depending on the class constants of $\alpha$ and $\beta$ only provided that

$$
\int_{0}^{1} \lg ^{m}(1 / t) \omega_{r^{\prime}}(t) d t / t<\infty \text {. }
$$

Proof. We begin with (4.15). Let $Q=\left\{y:\left|y-x_{0}\right|<d\right\}$. We have

$$
\begin{aligned}
& \int\|k(x, y)-k(z, y)\|\|f(y)\| d y \\
& =\sum_{k=3}^{\infty} \int_{2^{k} Q \sim 2^{k-1} Q}\|k(x, y)-k(z, y)\|\|f(y)\| d y .
\end{aligned}
$$

Since for $R=2^{k-1} d$ we have $2^{k} Q \sim 2^{k-1} Q=\left\{y: R \leq\left|y-x_{0}\right|<2 R\right\}$ and $\left|x-x_{0}\right|<d<R / 2,\left|z-x_{0}\right|<d<R / 2$, by Hölder inequality, and condition

(2.3) we get that the integral under the summation sign is bounded by

$$
\begin{gathered}
\left(\int_{2^{k} Q \sim 2^{k-1} Q}\|k(x, y)-k(z, y)\|^{r^{\prime}} d y\right)^{1 / r^{\prime}}\left(\int_{2^{k} Q}\|f(y)\|^{r} d y\right)^{1 / r} \\
\leq c\left(2^{-k}+\int_{|x-z| / 2^{k} d<t<|x-z| / 2^{k-1} d} \omega_{r^{\prime}}(t) d t / t\right) \\
\cdot\left(\int\|f(y)\|^{\lambda} \alpha(y)^{\lambda} d y\right)^{1 / \lambda}\left(\left|2^{k} Q\right|^{-1} \int_{2^{k} Q} \alpha^{-r(\lambda / r)^{\prime}}\right)^{1 / r(\lambda / r)^{\prime}} .
\end{gathered}
$$

Since $\alpha^{r} \in A(\lambda / r, \infty)$, the second member of (4.17) is bounded by

$$
c\left(\inf _{Q} \alpha^{-1}\right)\left(1+\int_{0}^{1} \omega_{r^{\prime}}(t) d t / t\right)\left(\int\|f(y)\|^{\lambda} \alpha(y)^{\lambda} d y\right)^{1 / \lambda} .
$$


Then, by Lemma (4.1), the expression (4.17) is less than or equal to

$$
\begin{aligned}
& c|Q|^{-1} \int_{Q}\left\|\tilde{a}(x)-\tilde{a}_{Q}\right\|^{m} \alpha(x)^{-1} d x \\
& \quad \leq c\left(|Q|^{-1} \int\left\|\tilde{a}(x)-\tilde{a}_{Q}\right\|^{m r(\lambda / r)^{\prime} v} \alpha(x)^{-r(\lambda / r)^{\prime} v} d x\right)^{1 / r(\lambda / r)^{\prime} v}\|\| f\left\|_{\alpha}\right\|_{\lambda} \\
& \quad \leq c\left(\inf _{Q} \beta^{-1}\right)\left(\int\|f(y)\|^{\lambda} \alpha(y)^{\lambda} d y\right)^{1 / \lambda},
\end{aligned}
$$

which proves $(4.15)$.

Let us prove (4.16). We have

$$
\begin{aligned}
& \int\|k(x, y)-k(z, y)\|\left\|a(y)-a_{Q}\right\|^{m}\|f(y)\| d y \\
& =\sum_{k=3}^{\infty} \int_{2^{k} Q \sim 2^{k-1} Q}\|k(x, y)-k(z, y)\|\left\|a(y)-a_{Q}\right\|^{m}\|f(y)\| d y .
\end{aligned}
$$

Since $\left\|a(y)-a_{Q}\right\| \leq\left\|a(y)-a_{2} k_{Q}\right\|+\left\|a_{2} k_{Q}-a_{Q}\right\|$ the integral under the summation sign is bounded by

$$
\begin{aligned}
& c \int_{2^{k} Q \sim 2^{k-1} Q}\|k(x, y)-k(z, y)\|\left\|a(y)-a_{2} k_{Q}\right\|^{m}\|f(y)\| d y \\
& \quad+c\left\|a_{2} k_{Q}-a_{Q}\right\|^{m} \int_{2^{k} Q \sim 2^{k-1} Q}\|k(x, y)-k(z, y)\|\|f(y)\| d y=A_{k}+B_{k} .
\end{aligned}
$$

As for $A_{k}$, by Lemma (4.1) we get

$$
\begin{aligned}
A_{k} \leq c & \left(\int_{2^{k} Q 2^{k-1} Q}\|k(x, y)-k(z, y)\|^{r^{\prime}} d y\right)^{1 / r^{\prime}} \\
& \cdot\left(\int_{2^{k} Q}\left\|a(y)-a_{2} k_{Q}\right\|^{m r(\lambda / r)^{\prime}} \alpha(y)^{-r(\lambda / r)^{\prime}} d y\right)^{1 / r(\lambda / r)^{\prime}} \\
& \cdot\left(\int\|f(y)\|^{\lambda} \alpha(y)^{\lambda} d y\right)^{1 / \lambda} c\left(2^{k} d\right)^{-1 / r(\lambda / r)^{\prime}} \\
\leq & \left(2^{-k}+\int_{|x-z| / 2^{k} d<t<|x-z| / 2^{k-1} d} \omega(t) d t / t\right) \\
& \cdot\left(\inf _{Q} \beta^{-1}\right)\left(\int\|f(y)\|^{\lambda} \alpha(y)^{\lambda} d y\right)^{1 / \lambda} .
\end{aligned}
$$

In order to estimate $B_{k}$, applying Hölder inequality twice, we obtain

$$
\begin{aligned}
B_{k} \leq & \left\|a_{2} k_{Q}-a_{Q}\right\|^{m}\left(\int_{2^{k} Q \sim 2^{k-1} Q}\|k(x, y)-k(z, y)\|^{r^{\prime}} d y\right)^{1 / r^{\prime}} \\
& \cdot\left(\int_{2^{k} Q} \alpha(x)^{r(\lambda / r)^{\prime}} d x\right)^{1 / r(\lambda / r)^{\prime}}\left(\int\|f(y)\|^{\lambda} \alpha(y)^{\lambda} d y\right)^{1 / \lambda} .
\end{aligned}
$$

Since $\left\|a_{2} k_{Q}-a_{Q}\right\| \leq \sum_{j=1}^{k}\left\|a_{2} j_{Q}-a_{2^{j-1}} Q\right\|$ and

$$
\left\|a_{2} j_{Q}-a_{2^{j-1}}\right\| \leq 2^{n}\left|2^{j} Q\right|^{-1} \int_{2^{j} Q}\left\|a(y)-a_{2} j_{Q}\right\| d y,
$$


we get

$$
\begin{aligned}
& \left\|a_{2} k_{Q}-a_{Q}\right\|^{m}\left(\int_{2^{k} Q} \alpha(x)^{-r(\lambda / r)^{\prime}} d x\right)^{1 / r(\lambda / r)^{\prime}} \\
& \quad \leq c\left\|a_{2} k_{Q}-a_{Q}\right\|^{m}\left(\inf _{2^{k} Q} \alpha^{-1}\right)\left|2^{k} Q\right|^{1 / r(\lambda / r)^{\prime}} \\
& \quad \leq c k^{m}\left|2^{k} Q\right|^{1 / r(\lambda / r)^{\prime}} \max _{1 \leq j \leq k}\left(\left|2^{j} Q\right|^{-1} \int_{2^{j} Q}\left\|a(y)-a_{2} j_{Q}\right\|^{m r(\lambda / r)^{\prime}} \alpha(y)^{-r(\lambda / r)^{\prime}} d y\right)^{1 / r(\lambda / r)^{\prime}} \\
& \quad \leq c k^{m}\left(\inf _{Q} \beta^{-1}\right)\left|2^{k} Q\right|^{1 / r(\lambda / r)^{\prime}} .
\end{aligned}
$$

Therefore, by condition (2.3),

$$
\begin{aligned}
B_{k} \leq & c\left(\inf _{Q} \beta^{-1}\right) k^{m}\left(2^{-k}+\int_{|x-z| / 2^{k} d<t<|x-z| / 2^{k-1} d} \omega(t) d t / t\right) \\
& \cdot\left(\int\|f(y)\|^{\lambda} \alpha(y)^{\lambda} d y\right)^{1 / \lambda} .
\end{aligned}
$$

Adding up our estimates on $A_{k}$ and $B_{k}$, we get that (4.16) is bounded by

$$
c\left(\inf _{Q} \beta^{-1}\right)\left(1+\int_{0}^{1} \lg ^{m}(1 / t) \omega_{r^{\prime}}(t) d t / t\right)\left(\int\|f(y)\|^{\lambda} \alpha(y)^{\lambda} d y\right)^{1 / \lambda},
$$

as we wanted to show. The proofs of $\left(4.15^{\prime}\right)$ and $\left(4.16^{\prime}\right)$ are just the same.

Proofs of Theorems (2.1) and (2.4). Let us prove Theorem (2.4) first. Let us estimate $\left(W_{m} f\right)^{\#}\left(x_{0}\right)$. Let $Q$ be a ball and $x_{0} \in Q$. Let us assume, as we may, that $a_{Q}=0$. Let $f=f_{1}+f_{2}$, where $f_{1}=f \chi_{4 Q}$. We define $c_{Q} \in F$ as

$$
c_{Q}=|Q|^{-1} \int_{Q}\left(\int|a(y)|^{m} w(z, y) f_{2}(y) d y\right) d z .
$$

Then,

$$
\begin{aligned}
|Q|^{-1} & \int_{Q}\left\|W_{m} f(x)-c_{Q}\right\| d x \\
& \leq|Q|^{-1} \int_{Q}\left\|W_{m} f_{1}(x)\right\| d x+|Q|^{-1} \int_{Q}\left\|W_{m} f_{2}(x)-c_{Q}\right\| d x \\
& =A_{1}+A_{2} .
\end{aligned}
$$

For $A_{1}$, we have

$$
A_{1} \leq c|Q|^{-1} \int_{Q}|a(x)|^{m}\left\|W\left(\left|f_{1}\right|\right)(x)\right\| d x+c|Q|^{-1} \int_{Q}\left\|W\left(|a|^{m}\left|f_{1}\right|\right)\right\| d x
$$

As for $A_{2}$, recalling that for $v \in F,\|(|v|)\|=\|v\|$, we have

$$
A_{2} \leq|Q|^{-1} \int_{Q}\left\||Q|^{-1} \int_{Q}\left(\int\left|(a(y)-a(x))^{m} w(x, y)-a(y)^{m} w(z, y)\right|\left|f_{2}(y)\right| d y\right) d z\right\| d x .
$$


Let us estimate the expression inside the norm. We have

$$
\begin{aligned}
|Q|^{-1} & \int_{Q}\left(\int\left|(a(y)-a(x))^{m} w(x, y)-a(y)^{m} w(z, y)\right|\left|f_{2}(y)\right| d y\right) d z \\
\leq & \sum_{j=0}^{m-1}|a(x)|^{m-j} \int w(x, y)|a(y)|^{j}\left|f_{2}(y)\right| d y \\
& +|Q|^{-1} \int_{Q}\left(\int|w(x, y)-w(z, y)||a(y)|^{m}\left|f_{2}(y)\right| d y\right) d z=B_{1}+B_{2} .
\end{aligned}
$$

Since $|a(y)|^{i} \leq|Q|^{-1} \int_{Q}|a(y)-a(z)|^{i} d z$, we get

$$
\begin{aligned}
B_{1} \leq & c \sum_{i=0}^{m-1}|a(x)|^{m-j}|Q|^{-1} \int_{Q}\left(\int|a(y)-a(z)|^{i}|w(x, y)-w(z, y)|\left|f_{2}(y)\right| d y\right) d z \\
& +\sum_{i=0}^{m-1}|a(x)|^{m-j}|Q|^{-1} \int_{Q} W_{i}(|f|)(z) d z=B_{11}+B_{12}
\end{aligned}
$$

and

$\left.B_{11} \leq c|Q|^{-1} \int_{Q} \int\left(|a(x)|^{m}+|a(y)|^{m}+|a(z)|^{m}\right)|w(x, y)-w(z, y)|\left|f_{2}(y)\right| d y\right) d z$.

Thus, collecting estimates, and dropping the assumption $a_{Q}=0$, we obtain that

$$
\begin{aligned}
& |Q|^{-1} \int_{Q}\left\|W_{m} f(x)-c_{Q}\right\| d x \\
& \leq c\left\{|Q|^{-1} \int_{Q}\left|a(x)-a_{Q}\right|^{m}\left\|W\left(\left|f_{1}\right|\right)(x)\right\| d x+|Q|^{-1}\right. \\
& \quad \cdot \int_{Q}\left\|W\left(\left|a-a_{Q}\right|^{m}\left|f_{1}\right|\right)(x)\right\| d x \\
& \quad+|Q|^{-1} \int_{Q}\left|a(x)-a_{Q}\right|^{m} \int\|w(x, y)-w(z, y)\|\left|f_{2}(y)\right| d y \\
& \left.\quad+|Q|^{-1} \int_{Q} \int\|w(x, y)-w(z, y)\|\left|a(y)-a_{Q}\right|^{m}\left|f_{2}(y)\right| d y\right\} \\
& \quad+c \sum_{i=0}^{m-1}\left(|Q|^{-1} \int_{Q}\left|a(x)-a_{Q}\right|^{m-i} d x\right)\left(|Q|^{-1} \int_{Q} W_{i}(|f|)(z) d z\right) \\
& =c R_{m}(f, Q)+c \sum_{i=0}^{m-1} S_{m, i}\left(W_{i}(|f|), Q\right),
\end{aligned}
$$

where $R_{m}(f, Q)$ is the expression inside the brackets and

$$
S_{m, i}(g, Q)=\left(|Q|^{-1} \int_{Q}\left|a(x)-a_{Q}\right|^{m-i} d x\right)\left(|Q|^{-1} \int_{Q}|g(z)| d z\right) .
$$

If we define $R_{m}(f)\left(x_{0}\right)=\sup _{x_{0} \in Q} R_{m}(f, Q)$ and

$$
S_{m, i}(g)\left(x_{0}\right)=\sup _{x_{0} \in Q} S_{m, i}(g, Q),
$$


we get

$$
W_{m}(f)^{\#}\left(x_{0}\right) \leq c\left[R_{m}(f)\left(x_{0}\right)+\sum_{i=0}^{m-1} S_{m, i}\left(W_{i}(|f|)\right)\left(x_{0}\right)\right] .
$$

Thus, by Lemmas (4.6), (4.9) and (4.14), we get

$$
\left\|\beta R_{m} f\right\|_{\infty} \leq c\left(\int|f(x)|^{\lambda} \alpha(x)^{\lambda} d x\right)^{1 / \lambda},
$$

provided that $\nu^{m}=\alpha \beta^{-1}, \alpha^{r}$ and $\beta^{r}$ belonging to $A\left(\left((\lambda / r)^{\prime} v\right)^{\prime}, \infty\right), v$ any number greater than one and the constant $c$ depending only on the class constants of $\alpha$ and $\beta$.

Also, by Lemma (4.12) we get $\left\|\beta S_{m, i}(g)\right\|_{\infty} \leq c\|\alpha g\|_{\infty}$, provided that $\alpha \beta^{-1}=\nu^{m-i}, \alpha$ and $\beta$ belonging to $A\left(v^{\prime}, \infty\right), v$ any number greater than one and $c$ depending on the class constants of $\alpha$ and $\beta$.

Let $T_{0}(f)=R_{m}\left(f \nu^{-m}\right)$. Then

$$
\begin{aligned}
\left\|\beta T_{0} f\right\|_{\infty} & =\left\|\beta R_{m}\left(f \nu^{-m}\right)\right\|_{\infty} \leq c\left(\int|f|^{\lambda} \nu^{-m \lambda} \alpha^{\lambda} d x\right)^{1 / \lambda} \\
& =c\left(\int|f|^{\lambda} \beta^{\lambda} d x\right)^{1 / \lambda}
\end{aligned}
$$

whenever $\beta^{r}$ and $\left(\nu^{m} \beta\right)^{r}$ belong to $A\left(\left((\lambda / r)^{\prime} v\right)^{\prime}, \infty\right)$.

By the Extrapolation Theorem (1.3) we get that $\left\|\beta T_{0} f\right\|_{q} \leq c\|\beta f\|_{p}$ holds for $\beta^{r}$ and $\left(\nu^{m} \beta\right)^{r}$ belonging to $A\left(\left((p / r)^{\prime} t\right)^{\prime}, q\right), q^{-1}=p^{-1}-\lambda^{-1}$. Thus $\left\|\beta R_{m}(f)\right\|_{q} \leq c\|\alpha f\|_{p}$, provided $\alpha \beta^{-1}=\nu^{m}$.

Let $T_{i}(g)=S_{m, i}\left(g \nu^{i-m}\right)$. Then by Lemma (4.12), we have

$$
\left\|\beta T_{i} g\right\|_{\infty}=\left\|\beta S_{m, i}\left(g \nu^{i-m}\right)\right\|_{\infty} \leq c\left\|g \nu^{i-m} \delta\right\|_{\infty}=c\|g \beta\|_{\infty}
$$

whenever $\beta$ and $\delta=\nu^{m-i} \beta$ belong to $A\left(v^{\prime}, \infty\right)$. Then by the Extrapolation Theorem (1.3), in the case $\lambda=\infty$, we get $\left\|\beta T_{i} g\right\|_{q} \leq c\|\beta g\|_{q}$ for every $\beta$ such that $\beta^{r}$ and $\left(\nu^{m-i} \beta\right)^{r}$ belong to $A\left(\left((q / r)^{\prime} t\right)^{\prime}, q / r\right)$. Therefore, if $\delta_{i}$ is the weight given in Lemma (4.5) and observing that since $p \leq q$, $A\left(\left((q / r)^{\prime} t\right)^{\prime}, q / r\right) \subset A\left(\left((p / r)^{\prime} t\right)^{\prime}, q / r\right)$, we have that

$$
\begin{aligned}
& \left\|\beta S_{m, i}\left(W_{i} f\right)\right\|_{q}=\left\|\beta T_{i}\left(\nu^{m-i} W_{i}(f)\right)\right\|_{q} \\
& \quad \leq c\left\|\beta \nu^{m-i} W_{i}(f)\right\|_{q}=c\left\|\delta_{i} W_{i}(f)\right\|_{q} \leq c\|\alpha f\|_{p}
\end{aligned}
$$

holds. Thus, by (4.18), we obtain

$$
\left\|\beta\left(W_{m} f\right)^{\#}\right\|_{q} \leq c\left\|\beta R_{m}(f)\right\|_{q}+c \sum_{i=0}^{m-1}\left\|\beta S_{m, i}\left(W_{i} f\right)\right\|_{q} \leq c\|\alpha f\|_{p},
$$

as we wanted to show.

In order to finish the proof of Theorem (2.4) we have to show that the sharp function theorem, see [CF and RFC], applies. Let $f \in C_{0}^{\infty}$ and let $Q$ be any ball containing the support of $f$. Then, the function $W_{m} f(x)$ is well defined and belongs to $L^{(1+\varepsilon)}$ on $Q$. In fact,

$$
\left\|W_{m} f(x)\right\| \leq c\left|a(x)-a_{Q}\right|^{m}\|W(|f|)(x)\|+c\left\|W\left(\left|a-a_{Q}\right||f|\right)(x)\right\| .
$$


Then,

$$
\begin{aligned}
\int_{Q}\left\|W_{m} f(x)\right\|^{1+\varepsilon} d x \leq c\left(\int_{Q}\left|a-a_{Q}\right|^{m(1+\varepsilon) s} d x\right)^{1 / s} \\
\cdot\left(\int\|W(|f|)(x)\|^{(1+\varepsilon) s^{\prime}} d x\right)^{1 / s^{\prime}} \\
+|Q|^{1 / s}\left(\int_{Q}\left\|W\left(\left|a-a_{Q}\right|^{m}|f|\right)(x)\right\|^{(1+\varepsilon) s^{\prime}} d x\right)^{1 / s^{\prime}} .
\end{aligned}
$$

If $s$ and $\varepsilon$ are chosen so that $(1+\varepsilon) s$ is close enough to zero, then, by Lemma (4.4)

$$
\left(\int_{Q}\left|a-a_{Q}\right|^{m(1+\varepsilon) s} d x\right)^{1 / s}<\infty .
$$

Moreover, since $(1+\varepsilon) s^{\prime}$ can be assumed to be greater than $\lambda^{\prime}$, by the boundedness of the operator $W$ and Lemma (4.4) again, the second member of (4.19) is seen to be finite. Let $M_{Q}$ be the Hardy-Littlewood maximal function restricted to cubes contained in $Q$ and analogously $f^{\# Q}$ the sharp function obtained by using the same cubes. Then, if $0<\eta$ is small enough we have

$$
\begin{aligned}
\int_{Q} \beta^{q} M_{Q}(\|W f\|)^{\eta} d x \leq & \left(\int_{Q} M_{Q}(\|W f\|)^{1+\varepsilon} d x\right)^{\eta / 1+\varepsilon} \\
& \cdot\left(\int_{Q} \beta^{q((1+\varepsilon) / \eta)^{\prime}} d x\right)^{1 /\left((1+\varepsilon) / \eta^{\prime}\right)^{\prime}} .
\end{aligned}
$$

Since $\eta$ is as small as we please, $[(1+\varepsilon) / \eta]^{\prime}$ is sufficiently close to one, therefore, by the maximal theorem we see that the second member of $(4.20)$ is finite. Then, by the sharp function theorem we have

$$
\begin{aligned}
\int_{Q}\|W f\|^{q} \beta^{q} d x & \leq c \int_{Q}\left(\|W f\|^{\#, Q} \beta\right)^{q} d x \\
& \leq c \int_{Q}\left((W f)^{\#} \beta\right)^{q} d x \leq c \int\left((W f)^{\#} \beta\right)^{q} d x,
\end{aligned}
$$

where the constant depends on $\beta$ only. Thus, enlarging $Q$ we get

$$
\left(\int\|W f\|^{q} \beta^{q} d x\right)^{1 / q} \leq c\left(\int\left((W f)^{\#}\right)^{q} \beta^{q} d x\right)^{1 / q} \leq c\left(\int|f|^{p} \alpha^{p} d x\right)^{1 / p}
$$

as we wanted to show.

Proof of Theorem (2.1). Let $Q$ be a ball. By conditions (i) and (iii) of Theorem (2.1), it follows easily by induction on $m$ that

$$
C^{m}\left(a_{Q} f\right)=\tilde{a}_{Q} C^{m}(f), \quad C_{a, \tilde{a}}^{m}(f)=C_{a-a_{Q}, \tilde{a}-\tilde{a}_{Q}}^{m}(f),
$$

and

$$
C^{m}(f)(x)=\sum_{k=0}^{m}(-1)^{k}\left(\begin{array}{c}
k \\
m
\end{array}\right) \tilde{a}(x)^{m-k} K\left(a^{k} f\right)(x)
$$


By (4.21), we can assume that $a_{Q}=0$. By elimination, from (4.22) we get that for suitable constants $r_{k}$, we have

$$
C^{m}(f)=\sum_{0}^{m-1} r_{k} \tilde{a}^{m-k} C^{k} f+(-1)^{m} K\left(a^{m} f\right) .
$$

Let $f=f_{1}+f_{2}, f_{1}=f \cdot \chi_{4 Q}$. We define $c_{Q}$ as

$$
\begin{aligned}
c_{Q} & =(-1)^{m}|Q|^{-1} \int_{Q} K\left(a^{m} f_{2}\right)(z) d z \\
& =(-1)^{m}|Q|^{-1} \int_{Q}\left(\int k(z, y) a(y)^{m} f_{2}(y) d y\right) d z .
\end{aligned}
$$

The vector $c_{Q}$ is well defined if $f$ has a compact support. Then,

$$
\begin{aligned}
C^{m}(f)(x)-c_{Q}= & \left(\sum_{k=0}^{m-1} r_{k} \tilde{a}(x)^{m-k} C^{k}(f)(x)\right)+(-1)^{m} K\left(a^{m} f_{1}\right)(x) \\
& +\left((-1)^{m} K\left(a^{m} f_{2}\right)(x)-c_{Q}\right)
\end{aligned}
$$

Since, for $x \in Q$, we have

$$
\begin{aligned}
& (-1)^{m} K\left(a^{m} f_{2}\right)(x)-(-1)^{m}|Q|^{-1} \int_{Q} K\left(a^{m} f_{2}\right)(z) d z \\
& =(-1)^{m}|Q|^{-1} \int_{Q} \int[k(x, y)-k(z, y)] a^{m}(y) f_{2}(y) d y d z
\end{aligned}
$$

we get (dropping the assumption $a_{Q}=0$ )

$$
\begin{aligned}
& |Q|^{-1} \int_{Q}\left\|C^{m} f(x)-c_{Q}\right\| d x \\
& \leq c \sum_{k=0}^{m-1}|Q|^{-1} \int_{Q}\left\|\tilde{a}(x)-\tilde{a}_{Q}\right\|^{m-k}\left\|C^{k} f(x)\right\| d x \\
& \quad+c\left\{|Q|^{-1} \int_{Q}\left\|K\left(\left(a-a_{Q}\right)^{m} f_{1}\right)(x)\right\| d x\right. \\
& \left.\quad+|Q|^{-1} \int_{Q}\left(\int\|k(x, y)-k(z, y)\|\left\|a(x)-a_{Q}\right\|^{m}\left\|f_{2}(y)\right\| d y\right) d z\right\} \\
& =c\left\{\sum_{k=0}^{m-1} R_{k}\left(C^{k} f, Q\right)+S(f, Q)\right\},
\end{aligned}
$$

where $R_{k}(g, Q)=|Q|^{-1} \int_{Q}\left\|\tilde{a}(x)-a_{Q}\right\|^{m-k}\|g\| d x$.

Thus, if $R_{k}(g)\left(x_{0}\right)=\sup _{x_{0} \in Q} R_{k}(g, Q)$ and $S(f)\left(x_{0}\right)=\sup _{x_{0} \in Q} S(f, Q)$, we obtain the estimate

$$
C^{m}(f)^{\#}\left(x_{0}\right) \leq c\left\{\sum_{k=0}^{m-1} R_{k}\left(C^{k} f\right)\left(x_{0}\right)+S(f)\left(x_{0}\right)\right\} .
$$

From this estimate, the proof of the theorem is almost the same as that of Theorem (2.4) and will not be given. 


\section{REFERENCES}

[B] S. Bloom, A commutator theorem and weighted BMO, Trans. Amer. Math. Soc. 292 (1985), 103-122.

[Bu] D. L. Burkholder, A geometric condition that implies the existence of certain singular integrals of Banach-space-valued functions, Conference on Harmonic Analysis in Honor of Antoni Zygmund, University of Chicago, 1981, Wadsworth, Belmont, Calif., 1983.

[CH] S. Chanillo, A note on commutators, Indiana Univ. Math. J. 31 (1982), 7-16.

[CRW] R. Coifman, R. Rochberg and G. Weiss, Factorization theorems for Hardy spaces of several variables, Ann. of Math. (2) 103 (1976).

[CF] A. Córdoba and C. Fefferman, $A$ weighted norm inequality for singular integrals, Studia Math. 57 (1976), 97-101.

[RFC] J. García-Cuerva and J. L. Rubio de Francia, Weighted norm inequalities and related topics, Math. Studies 116, North-Holland, Amsterdam, 1985.

[HMS] E. Harboure, R. A. Macias and C. Segovia, Extrapolation results for classes of weights, Amer. J. Math. 110 (1988), 383-397.

[H] R. A. Hunt, On the convergence of Fourier series, orthogonal expansions and their continuous analogues, Proc. Conf. Edwardsville, Southern Illinois Univ. Press, 1968, pp. 235-255.

[KW] D. S. Kurtz and R. L. Wheeden, Results of weighted norm inequalities for multipliers, Trans. Amer. Math. Soc. 255 (1979), 343-362.

[Mu] B. Muckenhoupt, Weighted norm inequalities for the Hardy maximal function, Trans. Amer. Math. Soc. 165 (1972), 207-226.

[FRT] J. L. Rubio de Francia, F. J. Ruiz and J. L. Torrea, Calderón-Zygmund theory for operatorvalued kernels, Adv. in Math. 62 (1986), 7-48.

[RT] F. J. Ruiz and J. L. Torrea, Weighted and vector valued inequalities for potential operators, Trans. Amer. Math. Soc. 295 (1986), 213-232.

[STa] C. Segovia and J. L. Torrea, Extrapolation of pairs of related weights, Analysis and Partial Differential Equations: A Collection of Papers Dedicated to Misha Cotlar, Marcel Dekker, New York, 1990.

[STb] $\frac{}{971}$, Vector valued commutators and applications, Indiana Univ. Math. J. 38 (1989), 959-

[STW] J.-O. Strömberg and R. L. Wheeden, Fractional integral on weighted $H^{p}$ and $L^{p}$ spaces, Trans. Amer. Math. Soc. 287 (1985), 293-321.

IAM-CONICET and Universidad de Buenos Aires (FCEyN), Buenos Aires, Argentina

Current address: IAM, Viamonte 1636, 1055 Bs. As., Argentina

Departmento de Matematica, Universidad Autónoma de Madrid, 28049 Madrid, Spain 